\title{
LAS TEORÍAS IMPLÍCITAS DE PROFESORES UNIVERSITARIOS BRASILEÑOS ACERCA DE LA MOTIVACIÓN DE SUS ALUMNOS PARA APRENDER
}

\author{
Iron Pedreira Alves ${ }^{1}$ \\ Juan Ignacio Pozo ${ }^{2}$
}

\begin{abstract}
RESUMEN
La motivación para aprender es un proceso psicológico importante en todos los niveles de la educación formal. Por eso, es útil conocer qué piensan docentes y discentes sobre dicho proceso psicológico si se busca conseguir una enseñanza superior más efectiva. El presente estudio tuvo como objetivo analizar las teorías implícitas de profesores universitarios de cursos de formación de profesorado acerca de la motivación de sus alumnos para aprender. Se llevaron a cabo entrevistas semiestructuradas en profundidad con 32 profesores de universidades públicas y privadas del área de educación en Brasil. Las entrevistas fueron grabadas, transcritas y sometidas a un análisis de contenido, basado en el marco conceptual de las teorías implícitas sobre enseñanza y aprendizaje. Los resultados sugieren que los profesores adoptan en su mayoría la Teoría Interpretativa, lo que está de acuerdo con estudios anteriores. Además, las respuestas clasificadas como de Teoría Directa tienden a responsabilizar las características de los estudiantes, mientras que las de Teoría Interpretativa tienden a incluir el contexto de aprendizaje como factor influyente en la motivación. Estos datos, que se clarifican mediante la presentación de fragmentos de las respuestas, sugieren la necesidad de construir concepciones más elaboradas sobre la motivación.
\end{abstract}

Conceptos clave: teorías implícitas, docencia universitaria, motivación académica, concepciones del profesor, formación docente.

\section{THE IMPLICIT THEORIES OF BRAZILIAN UNIVERSITY TEACHERS ON THE MOTIVATION OF THEIR STUDENTS TO LEARN}

\section{ABSTRACT}

Motivation to learn is an important psychological process on all levels of formal education. Therefore, it is useful to know what teachers and students think about this psychological process if to achieve a more effective higher education is sought. The present study aimed to analyze the implicit theories of university professors of teacher training courses on the motivation of their students to learn. In-depth semi-structured interviews were carried out with 32 professors from

Universidade Estadual de Feira de Santana, Feira de Santana, Brasil. Contacto: ipalves@uefs.br

Universidad Autónoma de Madrid, Madrid, España. Contacto: nacho.pozo@uam.es 
public and private universities in the area of education in Brazil. The interviews were recorded, transcribed and subjected to content analysis, based on the conceptual framework of the implicit theories about teaching and learning. The results suggest that teachers mostly adopt interpretive theory, which is in agreement with previous studies. In addition, the responses classified as Direct Theory tend to hold the students' characteristics responsable, while those of the Interpretive Theory tend to include the learning context as an influencing factor in motivation. These data, which are clarified through the presentation of fragments of the answers, suggest the need to build more elaborate conceptions about motivation.

Key concepts: implicit theories, university teaching, academic motivation, teacher conceptions, teacher training. 


\section{Introducción}

De acuerdo con la Real Academia Española (RAE), la motivación "es un ensayo mental preparatorio de una acción para animarse a ejecutarla con interés y diligencia". Huertas (2006) resalta dos aspectos que cree importantes en esta definición: primero, que se debe entender la motivación como un proceso psicológico y no meramente cognitivo, lo que significa que no se debe olvidar sus componentes afectivos y emocionales. Segundo, el autor advierte que solo se puede aplicar el concepto de "motivación" a los comportamientos humanos que tienen algún grado de voluntariedad.

A su vez, Schunk, Meece y Pintrich (2014, p. 5) afirman que "la motivación es el proceso mediante el cual las actividades dirigidas a un objetivo son instigadas y sostenidas". Pero la motivación de las personas no es accesible directamente, ni siquiera por ellas mismas, algo muy relevante para la comprensión y análisis de los datos de este estudio: "Como proceso, no observamos la motivación directamente, sino que inferimos su presencia a partir de acciones (por ejemplo, elección de tareas, esfuerzo, persistencia) y verbalizaciones (por ejemplo, 'realmente quiero trabajar en esto')" (Schunk, Meece \& Pintrich, 2014, p. 5). De acuerdo con esta idea — que inferimos la motivación de alguien de manera indirecta, a partir de la observación de determinadas conductas-, Alonso-Tapia (2005) afirma que pensamos que una persona está motivada cuando presenta indicadores de interés, esfuerzo, concentración y satisfacción, y si estos indicadores están presentes de modo más o menos regular a lo largo de los cursos escolares.

Lo expuesto es doblemente importante para el presente estudio, pues los docentes no pueden acceder directamente a los estados motivacionales de sus estudiantes, sino inferirlos a partir de las conductas que estos manifiestan. De la misma manera, solo podemos acceder a las concepciones docentes sobre la motivación - o mejor, a sus teorías implícitas, que son el foco de interés de esta investigación - de modo indirecto, dado que, como se explicará, son en su mayor parte implícitas. 
Otro aspecto que se debe tener en cuenta al analizar las distintas maneras en que los profesores piensan acerca de la motivación académica, más específicamente acerca de las teorías implícitas desde las que se representan a sus alumnos, es que la mayoría se encuentra a medio camino entre dos polos bastante distintos. De acuerdo con Alonso-Tapia (2005), hay cuatro elementos que deben servir como referentes para que se promueva un cambio motivacional. En primer lugar, en vez de pensar que la motivación es un problema de la voluntad del estudiante, es preciso tener presente que depende de la interacción entre el alumno y el entorno; segundo, en lugar de pensar que la motivación varía si se cambia un aspecto del entorno, se debe saber que cambia si se actúa sobre el clima de aprendizaje; tercero, en vez de pensar que la motivación es estática, se debe pensar que es dinámica, es decir, que varía si cambia el clima del entorno; cuarto y último, en lugar de pensar que la motivación se consigue con intervenciones puntuales, es preciso tener presente que requiere una intervención continua.

\section{Teorías implícitas acerca de la motivación como mediadora en la acción docente}

Sabemos que tanto profesores como alumnos universitarios poseen creencias y supuestos respecto de lo que es el conocimiento y cómo se adquiere, aunque no sean necesariamente capaces de verbalizarlos, ya que lo esencial de estas creencias y supuestos se encuentra implícito en la mente de las personas, como teorías más o menos elaboradas (Pozo, 2009), pero que conducirían las acciones de aprender y de enseñar. Según Errázuriz-Cruz,

las teorías implícitas son constructos de pensamiento que intervienen en el conocimiento, debido a que son concepciones epistémicas de representación de la realidad que orientan la conducta de las personas e irradian el influjo de distintas visiones de mundo. Así, las concepciones que los sujetos ostentan acerca de un fenómeno conforman el conjunto de conocimientos que se estimulan mediante las demandas del entorno. (2020, p. 6) 
A su vez, Pérez Echeverría et al. (2006) presentan las teorías implícitas como uno de los enfoques que estudian las concepciones de aprendizaje y enseñanza. Según estas autoras, las referidas teorías son entendidas "como un conjunto de principios que restringen tanto nuestra forma de afrontar como de interpretar o atender las distintas situaciones de enseñanza-aprendizaje a las que nos enfrentamos" (Pérez Echeverría et al., 2006, p. 79).

Estas teorías son llamadas "implícitas", pues, a pesar de dirigir las acciones que responden a las situaciones de nuestra vida cotidiana, son realizadas sin pasar por un proceso explícitamente consciente. Son, en verdad, resultados de la experiencia personal frente al ambiente cultural del aprendizaje, es algo que sentimos, vivimos y experimentamos, y por eso es difícil compartirlas o modificarlas. De las teorías implícitas sobre aprendizaje, categorizadas por Pozo y colaboradores (Pozo y Scheuer, 1999; Pozo et al., 2006), se utilizarán como marco teórico en el presente estudio la Teoría Directa, la Teoría Interpretativa y la Teoría Constructiva. En lo que sigue se presentarán las definiciones generales de cada teoría implícita, así como sus características específicas en el ámbito de la motivación.

En la Teoría Directa hay una expectativa de reproducción del conocimiento, tanto por parte de quien enseña como por parte de quien aprende, ya que el conocimiento debe reflejar, como un espejo, la realidad. Por lo tanto, sin aceptar la idea de aproximaciones sucesivas a una comprensión de la realidad, esta forma de concebir el conocimiento entiende que el saber es una cuestión de todo o nada: o se conoce la realidad o no se la conoce. Solo existen las opciones de verdadero y falso, pues esta perspectiva "se basa en una epistemología realista ingenua de acuerdo con la cual la simple exposición al contenido u objeto del aprendizaje garantiza el resultado, concebido como una reproducción fiel de la información o modelo presentado" (Pozo et al., 2006, p. 120).

De esta definición general sobre la Teoría Directa algunos autores destacan aspectos relacionados específicamente con el tema de la motivación (esto vale también para las otras dos teorías implícitas). Martín et al. (2006) afirman que en la Teoría Directa la motivación 
sería una condición previa para el aprendizaje, en la cual el profesor no puede intervenir. Pérez Echeverría et al. (2006) establecen que los profesores que mantienen esta teoría asumen que la motivación sería la causa personal del fracaso escolar, con independencia de otros procesos de enseñanza/aprendizaje que tienen su origen en el propio alumno, y por eso sería difícil de modificar en el aula. Por último, Torrado y Pozo (2006) afirman que, para este mismo grupo, la motivación sería una condición previa al aprendizaje que se debe mantener mediante una adecuada distribución de premios o castigos, generalmente en forma de evaluación.

La Teoría Interpretativa aparece como una fase de transición entre las teorías Directa y Constructiva. Aunque existan diferencias entre las teorías Directa e Interpretativa, Pozo y Scheuer (1999) afirman que entre ellas hay el presupuesto epistemológico común de que es posible tener acceso a una única verdad absoluta. Sin embargo, los partidarios de la Teoría Interpretativa asumen que el aprendizaje es algo que consume tiempo y demanda esfuerzo deliberado. Es la teoría implícita más frecuente en los espacios educativos ya investigados (Scheuer y Pozo, 2006).

Para la Teoría Interpretativa, la motivación está influida por determinadas condiciones, como los intereses de los alumnos o las ayudas ofrecidas. Hay una relación de causalidad lineal y un dualismo entre cognición y emoción (Martín et al., 2006). Los "profesores interpretativos" entienden la motivación como un proceso cognitivo mediador, de responsabilidad del alumno, pero que ellos pueden gestionar, haciendo el aprendizaje más agradable (Torrado y Pozo, 2006). Según Pérez Echeverría et al. (2006), en esta concepción la motivación tendría su origen en la actividad social, lo que permitiría su modificación mediante intervención didáctica.

Se puede distinguir la Teoría Directa de la Teoría Interpretativa sobre la motivación en que los profesores que adoptan esta última creen que es posible cambiar los estados motivacionales de sus alumnos por medio de las decisiones y acciones que el profesor lleva a cabo. Es por tanto el profesor quien tiene la responsabilidad de gestionar los estados y procesos motivacionales del alumno. 
Finalmente, la Teoría Constructiva asume la idea de construir conocimientos sobre la realidad (en lugar de extraer de ella esos conocimientos) y se diferencia de las teorías anteriores principalmente por los principios epistemológicos, admitiendo que pueden existir diferentes grados de acercamiento a la verdad sobre un mismo objeto de conocimiento y que su apropiación implica necesariamente una transformación del contenido que se aprende, así como del propio aprendiz.

Las teorías implícitas de los profesores sobre motivación para aprender consideradas constructivas son las que más se acercan a los hallazgos más recientes de la psicología (Pozo, 2008). En líneas generales, creen en la fuerza del aprendizaje como elemento motivador. Para estos profesores, el alumno necesita aprender para sentirse competente. Desde esta posición, aprender es intrínsecamente motivador (Martín et al., 2006). La promoción de la motivación sería un proceso en que el profesor debe ayudar el alumno a construir sus propias metas, llevándolo a asumir progresiva responsabilidad en su propio aprendizaje (Torrado y Pozo, 2006). Por fin, creen que el objetivo de la enseñanza es ayudar a formular metas de aprendizaje, siendo la principal fuente de motivación la sensación de cualificación (Pérez Echeverría et al., 2006).

Aunque en el análisis mencionado Alonso-Tapia (2005) no recurra a la terminología de las teorías implícitas (Directa, Interpretativa y Constructiva), se puede admitir que las primeras características de cada uno de los cuatro elementos comentados al final de la introducción describen la manera de pensar propia de la Teoría Directa, mientras que las últimas caracterizan de modo bastante apropiado a la Teoría Constructiva.

Esta investigación ha tenido como objetivo general conocer las teorías implícitas de profesores universitarios de cursos de formación docente sobre la motivación de sus alumnos para aprender en sus disciplinas. Los objetivos específicos fueron: (1) saber si había una predominancia de respuestas en alguna de las tres teorías implícitas (Directa, Interpretativa y Constructiva) y (2) conocer, en profundidad, la manera de pensar de los docentes en cada teoría implícita, por medio de las respuestas al guion de entrevista. 


\section{Metodología}

En este estudio, de tipo descriptivo, tras realizar un estudio piloto para garantizar que las preguntas serían comprensibles, se diseñó una entrevista en profundidad y semiestructurada, en la que el entrevistador dispuso de un guion básico de preguntas que se cumplió con todos los entrevistados. Tras cada respuesta, sin embargo, el entrevistador podía formular otras cuestiones, tratando de profundizar, aclarar, organizar o sintetizar el pensamiento del entrevistado.

El guion contenía 27 preguntas, distribuidas en cinco apartados temáticos, además de un apartado introductorio para efecto de "calentamiento" (rapport). Los temas explorados fueron: requisitos para aprender, organización de la enseñanza, motivación para aprender, dificultades en el aprendizaje y evaluación del aprendizaje. El apartado acerca de motivación, foco del presente estudio, se componía de cinco preguntas:

1. ¿Cómo evalúa usted el empeño de sus alumnos para aprender en su disciplina?

2. ¿Cuál es la causa de esta situación (del empeño relatado en la cuestión anterior)?

3. ¿En qué medida eso interfiere en el aprendizaje de los alumnos?

4. ¿En qué medida eso interfiere en su manera de enseñar?

5. ¿Ese escenario podría ser mejorado? ¿Cómo?

La entrevista se aplicó a un total de 32 profesores universitarios de cursos de formación docente, pertenecientes a facultades públicas y privadas del Estado de Bahía, en Brasil. A todos los entrevistados se les solicitó su consentimiento informado. Todas las entrevistas fueron realizadas por el primer autor de este trabajo, grabadas en audio y posteriormente transcritas.

\subsection{Sistema de análisis}

Las respuestas de los entrevistados se sometieron a un "análisis de contenido". Cada extracto considerado significativo, llamado "unidad de registro", siguiendo a Bardin (2002), fue clasificado de 
acuerdo con un sistema de categorías generado a través de dos "vías" complementarias: una primera categorización se generó por vía deductiva, es decir, se utilizaron categorías previas definidas desde el marco teórico. Sobre los resultados de esta clasificación se produjo una segunda categorización por vía inductiva, es decir, se extrajeron, de un primer análisis de los datos, otras categorías que no estaban previamente definidas teóricamente.

En la primera se ha buscado identificar, en las respuestas, las unidades de registro que estaban de acuerdo con las definiciones acerca de las teorías implícitas sobre el aprendizaje propuestas en el marco teórico de este trabajo. Así, las unidades identificadas fueron agrupadas como representantes de la Teoría Directa, Teoría Interpretativa o Teoría Constructiva según los criterios señalados.

La segunda categorización fue realizada en dos etapas, ambas por la vía inductiva. En la primera se agruparon los extractos de cada teoría por semejanza temática, es decir, los enunciados que trataban de un mismo tema se agrupaban bajo una rúbrica que daba nombre a esa categoría (por ejemplo, Alumno influye al profesor, Lectura, Carrera, etc.). Tras agrupar todos los extractos, teniendo también como criterio la semejanza temática entre las rúbricas, éstas se organizaron en categorías más amplias denominadas "factores" (relativos al alumno: personales y contextuales; relativos a la enseñanza: profesor y currículo).

Como resultado de este proceso se obtuvieron las categorías y subcategorías recogidas en la tabla 1. Las categorías más amplias hacen hincapié en dos grupos de factores: los relativos al alumno, que se dividen en personales y de contexto; y los relativos a la enseñanza, que se dividen en profesor y currículo. Solo en los factores relativos a la enseñanza con foco en el profesor hubo respuestas características de las tres teorías implícitas, y la única teoría que apareció identificada en los cuatro factores fue la Teoría Interpretativa. Las definiciones de los factores y de las respectivas categorías serán presentadas más adelante en el apartado de resultados cualitativos, ilustradas mediante respuestas dadas por los participantes. 
Tabla 1

Criterios de organización de las respuestas

\begin{tabular}{|c|c|c|c|}
\hline & Teoría Directa & Teoría Interpretativa & Teoría Constructiva \\
\hline $\begin{array}{l}\text { Factores relativos } \\
\text { al alumno } \\
\text { (personales) }\end{array}$ & $\begin{array}{l}\text { Alumno influye al } \\
\text { profesor } \\
\text { Lectura } \\
\text { Carrera } \\
\text { Compromiso } \\
\text { Motivación como } \\
\text { un rasgo personal }\end{array}$ & $\begin{array}{l}\text { Alumno influye al } \\
\text { profesor } \\
\text { Lectura } \\
\text { Carrera } \\
\text { Compromiso } \\
\text { Esfuerzo }\end{array}$ & \\
\hline $\begin{array}{l}\text { Factores relativos al } \\
\text { alumno (contexto) }\end{array}$ & $\begin{array}{l}\text { Condiciones } \\
\text { socioeconómicas }\end{array}$ & $\begin{array}{l}\text { Condiciones } \\
\text { socioeconómicas }\end{array}$ & \\
\hline $\begin{array}{l}\text { Factores relativos } \\
\text { a la enseñanza } \\
\text { (profesor) }\end{array}$ & Evaluación & $\begin{array}{l}\text { Evaluación } \\
\text { Profesor } \\
\text { Clase } \\
\text { Estrategias }\end{array}$ & Método \\
\hline $\begin{array}{l}\text { Factores relativos } \\
\text { a la enseñanza } \\
\text { (currículo) }\end{array}$ & & $\begin{array}{l}\text { Currículo } \\
\text { Estructura }\end{array}$ & Sentido \\
\hline
\end{tabular}

Fuente: Elaboración propia.

Una vez diseñado el sistema de categorías, se hizo un análisis interjueces para asegurar la adecuación de las clasificaciones. Para este análisis se invitó a cinco voluntarios. Los criterios para participar de esta actividad fueron: tener conocimiento sobre el área de educación y comprender tanto el castellano como el portugués escrito, ya que las respuestas estaban redactadas en este último idioma. Cuando no había un acuerdo igual o superior al 90\% entre los evaluadores, se revisaba la redacción de las categorías en desacuerdo y se reenviaban junto con las demás, pero con nuevos extractos hasta que se alcanzaba el mencionado índice.

Una vez validadas las categorías, se aplicaron a todas respuestas, y los resultados obtenidos se introdujeron en un software apropiado para análisis cuantitativo (SPSS 20); a partir de este software se ha realizado el análisis estadístico de los resultados cuantitativos, utilizándose la ANOVA de medidas repetidas.

\section{Resultados}

A continuación, se presentan los principales hallazgos, organizados en resultados cuantitativos y resultados cualitativos. 


\subsection{Resultados cuantitativos}

Tras la lectura de las transcripciones, tal como se ha relatado en la metodología, fueron identificadas entre las respuestas un total de 117 unidades de registro (Bardin, 2002), distribuidas entre las tres teorías implícitas. Es importante destacar que no todos los profesores produjeron al menos una unidad de registro en algunas subcategorías y que algunos produjeron más de una en la misma respuesta. Se realizó un ANOVA de medidas repetidas sobre la frecuencia de las referidas respuestas. En la tabla siguiente se presenta la media y la desviación típica de cada categoría de análisis adoptada en este trabajo, agrupada en función de las tres teorías implícitas.

Tabla 2

Respuestas a las cuestiones sobre motivación

\begin{tabular}{lll}
\hline & $M$ & $D T$ \\
\hline Directa & $1.00^{*}$ & 0.88 \\
Interpretativa & $2.38^{*}$ & 1.93 \\
Constructiva & $0.34^{*}$ & 0.97 \\
\hline
\end{tabular}

Nota. $* p<, 050$.

Fuente: Elaboración propia.

Como se puede ver en la tabla 2, las respuestas de los profesores fueron clasificadas en su mayoría como interpretativas (más que la suma de las otras dos categorías juntas), $M=2.38$. La segunda categoría más frecuente fue la Directa, $M=1.00$, seguida de la Constructiva, $M=0.34$. Todas las diferencias entre las medias presentadas fueron estadísticamente significativas. Entre las respuestas categorizadas como Directa e Interpretativa la diferencia fue de $p<0,005$. Entre la Interpretativa y la Constructiva, de $\mathrm{p}<0,001$; y entre la Directa y la Constructiva, de $\mathrm{p}<0,031$. La Teoría Interpretativa ha sido, por lo tanto, la más frecuente. El análisis cualitativo de las respuestas que presentaremos a continuación, pormenorizado a través de las dimensiones recogidas en la tabla 1 , nos permitirá comprender mejor el sentido de estos datos.

Además, con el objetivo de saber si existían relaciones entre los diferentes factores identificados en la tabla 1 de las tres teorías implícitas de las que partíamos, los datos fueron sometidos también a 
la prueba de Spearman, con el fin de comprobar si había correlaciones estadísticamente significativas entre esas teorías y cada uno de esos factores.

Como se puede ver en la tabla 3, hubo una correlación significativa entre los factores relativos al alumno, centrados en aspectos personales, y la Teoría Directa $(r=0,50, p<0,01)$. Entre estos mismos factores y la Teoría Interpretativa la correlación fue más moderada $\mathrm{r}=0,354,(p<0,05)$. Los factores relativos al alumno, con énfasis en su contexto, se correlacionaron moderadamente solo con la Teoría Interpretativa $(\mathrm{r}=0,355 ; \mathrm{p}<0,05)$. Por último, hubo otra relación bastante significativa entre los factores relativos a la enseñanza, con énfasis en lo que hace el profesor, y la Teoría Interpretativa $(p<0,01 ; r=0,659)$. No se encontró relación significativa entre la Teoría Constructiva y alguno de los factores.

Tabla 3

Correlación entre las respuestas agrupadas por factores y por teorías implícitas

\begin{tabular}{|c|c|c|c|c|}
\hline & & $\begin{array}{l}\text { Teoría } \\
\text { Directa }\end{array}$ & $\begin{array}{l}\text { Teoría } \\
\text { Interpretativa }\end{array}$ & Teoría \\
\hline \multirow{3}{*}{$\begin{array}{l}\text { Factores relativos } \\
\text { al alumno } \\
\text { (personales) }\end{array}$} & $\begin{array}{l}\text { Coeficiente de } \\
\text { correlación }\end{array}$ &, $500 * *$ &, $354^{*}$ &,- 229 \\
\hline & $\begin{array}{l}\text { Significancia } \\
\text { Bilateral }\end{array}$ &, 004 &, 047 & ,207 \\
\hline & $\mathrm{N}$ & 32 & 32 & 32 \\
\hline \multirow[t]{3}{*}{$\begin{array}{l}\text { Factores relativos al } \\
\text { alumno (contexto) }\end{array}$} & $\begin{array}{l}\text { Coeficiente de } \\
\text { correlación }\end{array}$ & ,133 &, $355^{*}$ &,- 274 \\
\hline & $\begin{array}{l}\text { Significancia } \\
\text { Bilateral }\end{array}$ &, 467 &, 046 & , 129 \\
\hline & $\mathrm{N}$ & 32 & 32 & 32 \\
\hline \multirow{3}{*}{$\begin{array}{l}\text { Factores relativos } \\
\text { a la enseñanza } \\
\text { (profesor) }\end{array}$} & $\begin{array}{l}\text { Coeficiente de } \\
\text { correlación }\end{array}$ &,- 176 &, $659 * *$ &, 188 \\
\hline & $\begin{array}{l}\text { Significancia } \\
\text { Bilateral }\end{array}$ &, 334 &, 001 & ,303 \\
\hline & $\mathrm{N}$ & 32 & 32 & 32 \\
\hline \multirow{3}{*}{$\begin{array}{l}\text { Factores relativos } \\
\text { a la enseñanza } \\
\text { (currículo) }\end{array}$} & $\begin{array}{l}\text { Coeficiente de } \\
\text { correlación }\end{array}$ &,- 138 & , 162 & ,209 \\
\hline & $\begin{array}{l}\text { Significancia } \\
\text { Bilateral }\end{array}$ &, 452 &, 377 &, 250 \\
\hline & $\mathrm{N}$ & 32 & 32 & 32 \\
\hline
\end{tabular}

Nota. $* * p<, 010 ; * p<, 050$

Fuente: Elaboración propia. 
Estos resultados parecen reforzar los presupuestos teóricos sintetizados al comienzo de este artículo. Tal como describieron Martín et al. (2006) y Pérez Echeverría et al. (2006), los participantes de esta investigación que adoptan una Teoría Directa sobre la motivación para aprender la relacionan sobre todo con rasgos personales o propios de los alumnos. Los que sostienen una Teoría Interpretativa sitúan el foco no solo en el alumno sino también en el contexto y en la acción docente, lo que confirma datos obtenidos en otros estudios (Torrado y Pozo, 2006).

No se encontraron relaciones estadísticamente significativas entre la Teoría Constructiva y ninguno de los factores, lo cual puede ser debido al escaso número de respuestas constructivas encontradas (solo 11 respuestas del total de 117 extractos identificados). Para entender mejor la relación entre cada una de estas teorías y los factores mencionados, es necesario no obstante profundizar en las respuestas mediante un análisis cualitativo de las mismas.

\subsection{Resultados cualitativos}

A continuación presentaremos y comentaremos los extractos más representativos de cada una de las subcategorías, agrupados en sus respectivas teorías implícitas.

\subsubsection{Resultados cualitativos de la Teoría Directa}

Las subcategorías de este apartado se ubican, en su mayoría, en el grupo de factores de carácter más personal relativos al alumno. Solamente una pertenece a los factores relativos al alumno, pero con origen en su contexto, y otra pertenece a los factores relacionados con la enseñanza y centrados en el profesor.

De esta distribución se puede inferir que, para quienes piensan la motivación a partir del marco de la Teoría Directa, las características personales de los alumnos son las responsables de que estén motivados o desmotivados. El entorno de este alumno contribuye poco a su grado de motivación y, cuando lo hace, se presenta más como obstáculo que como incentivo. El profesor poco puede hacer para transformar la desmotivación del alumno, salvo 
exigir más de él y darle premios o castigos por medio de la evaluación (Torrado y Pozo, 2006).

Factores relativos al alumno (personales)

En este grupo están ubicadas las subcategorías que se centran en las características personales de los alumnos como determinantes de su grado de motivación, las que serán presentadas y comentadas. Al final de cada definición se informará, entre paréntesis, la respectiva frecuencia de extractos de respuestas.

Alumno influye al profesor: El comportamiento del alumno, si es más o menos dedicado o interesado, puede motivar o desmotivar al profesor, que tan solo reacciona al comportamiento de sus estudiantes sin intentar cambiarlo (tres extractos).

En una revisión de 130 estudios, Han y Yin (2016) encontraron, en muchos de ellos, que el comportamiento de los estudiantes es un factor determinante en la motivación docente para enseñar. En esta categoría encontramos respuestas en las que los profesores afirman que cambian su motivación en función de la motivación presentada por sus alumnos. Así que estos profesores no parecen concebir la promoción de la motivación como una de las responsabilidades de la docencia. En lugar de ser ellos quienes piensan en situaciones de aprendizaje que aumenten el interés y el consecuente esfuerzo de los estudiantes, se limitan a responder a los estados motivacionales mayoritarios en sus aulas:

Ya he dicho: si veo que los alumnos tienen interés, si le están dedicando energía, eso va a estimularme también. (Docente 24)

Lectura: Los profesores reconocen que las dificultades con la lectura suelen ser un obstáculo en el desempeño de los alumnos, pero lo máximo que hacen para mejorar la competencia lectora es exigir más de sus estudiantes (cuatro extractos).

Los profesores que tuvieron sus respuestas ubicadas en esta categoría seguramente estarían entre aquellos que, según Mateos (2009), demostrarían sorpresa, o incluso escepticismo, ante la idea 
de enseñar a leer en la universidad. Las habilidades de lectura, aunque reconocidamente importantes para el pleno desarrollo de las competencias del alumno universitario, deben estar completamente dominadas por él y no forman parte de los retos docentes. Como máximo, aceptarían la idea errónea de que "lo que hay que hacer para aprender a leer es precisamente leer mucho” (Mateos, 2009, p. 116).

Por el hecho de percibir que al alumno no le gusta leer, he exigido poca lectura. Son las lecturas básicas; bueno, esa lectura básica, por percibir que al alumno no le ha gustado leer; creo que voy a cambiarlo un poco, creo que voy a cambiar; ponerle a leer, léelo de todas formas, hay que leer... Lee y apáñate. Lee. Lee porque se necesita leer. (Docente 2)

Carrera: La carrera que elige el alumno determina la cantidad de esfuerzo invertido en el aprendizaje de las asignaturas (cinco extractos).

Con frecuencia se asume que aquellos estudiantes que acceden a carreras de menor prestigio social y, por lo tanto, menos concurridas, no siempre lo hacen por interés por la futura profesión. Es probable que la hayan elegido por la relativa facilidad de ingresar en la universidad y así disfrutar de las consecuencias positivas que eso implica. En Brasil, los cursos universitarios de formación de profesores suelen recibir a estos alumnos que declaran su preferencia por otra carrera considerada de difícil acceso.

Aunque reconozcan que la elección de la carrera tendría consecuencias directas para la motivación de sus alumnos y que hay, incluso, alumnos que optan por ser profesores, las respuestas de este grupo demuestran que algunos profesores no creen posible intervenir sobre este factor para aumentar la motivación de sus estudiantes.

Y he observado también que la mayoría que ingresa en el curso de Pedagogía ha optado por el curso sin saber qué estaba haciendo, porque era más fácil ingresar. Todavía hay el tema de la feminización del magisterio, la mayoría ingresa porque es un curso eminentemente femenino, aún existe este perfil. He notado en esos dos últimos semestres que son madres de familia, no son profesoras en servicio, que ingresaron 
en el curso. Se han puesto mucho en esta condición y presentan este perfil muy diferenciado, ese curso. (Docente 18)

Compromiso: La relación que el alumno establece con el curso y con su propia formación es interpretada por el profesor como presencia o ausencia de compromiso y determina la cantidad de esfuerzo invertida para aprender (cuatro extractos).

Esta categoría está directamente relacionada con la siguiente, ya que aquí también parece concebirse la motivación como algo exclusivamente intrínseco, en el sentido que expresa Huertas (2006, p. 99): "Una acción está intrínsecamente motivada cuando lo que interesa es la propia actividad, que es un fin en sí misma, no un medio para otras metas". Se puede afirmar, sin grandes probabilidades de error, que estos profesores entienden las actividades de aprendizaje como "actividades que se llevan a cabo en ausencia de contingencia externa aparente, sin ninguna recompensa que la regule claramente" (Ibid.).

La principal diferencia es que en estos enunciados los profesores se refieren explícitamente a la idea de "compromiso", utilizándola para explicar la inversión de tiempo y esfuerzo que hacen sus estudiantes para aprender en sus disciplinas. Las semejanzas se centran en la supuesta estabilidad de este compromiso, lo que dejaría poco margen para que el docente pueda actuar con el objetivo de involucrar más a los alumnos en el proceso de consecución de los resultados planteados por los primeros.

¡El alumno comprometido contribuye mucho! El compromiso, como he dicho, los gestos de compromiso, contribuyen bastante. Por ejemplo, fuera de la clase hablo con los estudiantes, y hay estudiantes que no hablan conmigo. Entonces, percibo que los alumnos que hablan conmigo en clase son los alumnos que presentan un mejor desempeño. (Docente 2)

Motivación como un rasgo personal: La dedicación o el esfuerzo de los alumnos para aprender dependen de una motivación (identificada como interés, curiosidad, compromiso) que es percibida como un rasgo estable de los estudiantes, algo que deben "poseer", 
independientemente de lo que haga el profesor en clase (nueve extractos).

Estos docentes no creen que la motivación de los estudiantes puede mejorar como resultado de prácticas pedagógicas adecuadas, tal como proponen las teorías actuales sobre motivación (AlonsoTapia, Ruiz y Huertas, 2020). En lugar de evitar las expectativas negativas y ofrecer ayuda a los alumnos que lo necesiten, estos profesores atribuyen toda la responsabilidad a una disposición previa e inmutable de los propios alumnos:

¡Creo que interfiere totalmente! Interfiere totalmente, porque no se esfuerzan, nosotros percibimos que muchos no se esfuerzan, son pocos. En este grupo de cuarenta se puede sacar cinco y nosotros notamos... Cinco o seis alumnos que de verdad tienen interés, que están comprometidos y que, de verdad, hacen que ese proceso de aprendizaje contribuya a su formación como futuros profesores. (Docente 16)

Factores relativos al alumno (contexto)

En este apartado se encuentra la subcategoría "condiciones socioeconómicas", que se centra en la influencia del entorno del alumno en su motivación.

Condiciones socioeconómicas: Las condiciones socioeconómicas de los alumnos son interpretadas por los profesores como obstáculos en el aprendizaje y determinan el grado de esfuerzo para aprender en la asignatura (tres extractos).

De acuerdo con ello, una de las principales características de la Teoría Directa en el campo de la motivación es que aquellos profesores que piensan conforme esta teoría implícita no se creen muy capaces de cambiar los estados motivacionales de sus alumnos, aunque piensen que pueden mejorar el rendimiento mediante premios y castigos.

En este grupo, tres entrevistados se quejan principalmente de la falta de tiempo y del cansancio de sus alumnos, que provienen de la necesidad que estos tienen de trabajar, como elementos desmotivadores para el aprendizaje. 
Otra cosa que no solemos hablar es el tema del cansancio. Hay alumnos que no trabajan en el ámbito de la educación; sin embargo, a veces tienen un trabajo que es muy agotador durante el día y eso (...) disminuye su dedicación a la asignatura de modo general. (Docente 8)

Factores relativos a la enseñanza (profesor)

En esta subcategoría el centro de las explicaciones cambia desde las características de los alumnos, vistas más como estados que como procesos, hacia lo que hace el profesor en clase.

Evaluación: El esfuerzo y consecuente desempeño de los alumnos dependen de cómo el profesor evalúa y/o califica las evaluaciones (cuatro extractos).

Finalizando la serie de extractos caracterizados como representantes de la Teoría Directa, es posible que la categoría "evaluación" represente el pensamiento característico de esta concepción implícita: la idea de que se puede hacer trabajar más al alumno por medio de la forma en que se le evalúa. La evaluación sería, por lo tanto, el principal instrumento que tienen los profesores para promover la motivación de sus estudiantes.

Sin embargo, a diferencia de lo que proponen García y Martínez (2013), los docentes con respuestas ubicadas en esta categoría no parecen creer que la evaluación continua y formativa sea la mejor solución para motivar los alumnos, sino que prefieren los exámenes tradicionales:

El esfuerzo de los estudiantes, de modo general —creo que no solamente en mi asignatura-, depende de cómo los evalúas. Entonces, si exiges, discutes con ellos, sobre todo en los exámenes realizados en clase sin consulta, se esfuerzan más, porque no tienen madurez para dedicarse a los estudios, excepto si parte de una necesidad de urgencia, que es la de obtener notas. Entonces, en este momento, por el hecho de ser del grado, lo que les preocupa son las notas. No es que en los demás niveles no preocupen, pero en ese nivel preocupa más. Así, la nota, es decir, como se les evalúa, les fuerza a estudiar. (Docente 7) 


\subsubsection{Resultados cualitativos de la Teoría Interpretativa}

Las subcategorías ubicadas en este apartado están distribuidas de manera más o menos equilibrada entre los factores relativos al alumno y los relativos a la enseñanza (ver tabla 1), siendo que los primeros incluyen una subcategoría menos que los últimos (cinco contra seis).

Factores relativos al alumno (personales)

En este grupo están ubicadas las categorías centradas en las características personales de los alumnos como determinantes de su grado de motivación, pero que, a diferencia de la Teoría Directa, pueden ser influidas por el profesor.

Alumno influye al profesor: El comportamiento del alumno, si es más o menos dedicado o interesado, puede motivar o desmotivar al profesor. Pero, a diferencia de la Teoría Directa, en la Teoría Interpretativa el profesor busca medios para superar esta desmotivación (seis extractos).

En esta categoría se puede percibir claramente que el aspecto motivacional en el aula es una vía de doble sentido. El profesor puede motivar a sus alumnos, pero también es afectado por sus comportamientos durante las clases. Estos profesores parecen poseer un sentido de autoeficacia, que les ayuda a superar la posible influencia negativa de la falta de empeño y motivación por parte de sus estudiantes.

A veces nos da un cierto desánimo. A veces creo que siento, viene eso de la desilusión, de la función del docente en ese espacio, de esa manera. No obstante, tampoco es algo que me detenga. (...) Aunque sepamos que existen dificultades, que depende del grupo con el que trabajemos, eso es más fuerte o menos fuerte, pero, por mi parte, hay eso de querer enfrentarlo y llevarlo en serio. (Docente 11)

Lectura: El profesor entiende que la competencia lectora es importante para un mejor aprendizaje. Por eso se preocupa de ofrecer las condiciones más favorables para su desarrollo (cinco extractos). 
En general, la dificultad en lectura fue una queja bastante frecuente entre los entrevistados. Aunque los profesores que tienen una concepción más interpretativa de la motivación crean que se puede mejorar la lectura de sus alumnos mejorando las condiciones que estos tienen para leer, lo que dejan entrever en sus respuestas es que no saben cómo crear mejores condiciones de lectura.

Creo que, a veces, es la dificultad de tener estrategias de lectura, pero en eso también me gustaría invertir más... en cómo trabajar las estrategias de lectura, que esto, a veces creo que dificulta. (Docente 26)

Carrera: La elección de la carrera influye en la relación de los alumnos con el aprendizaje y con el rendimiento, pero eso se puede cambiar por medio de factores internos o externos al alumno (seis extractos).

En Brasil, el acceso a las universidades se suele dar por medio de dos exámenes nacionales, el llamado "vestibular" y el "ENEM" (Exame Nacional do Ensino Médio). Aquellos estudiantes que se creen menos competentes para aprobar en cursos más demandados eligen otros en los que la probabilidad de aprobar es más grande. Por desgracia, entre estos cursos seguramente se encuentran los de formación de profesores. Este hecho es considerado por muchos profesores como un elemento desmotivador con relación al aprendizaje, ya que a los estudiantes les gustaría estar en otra carrera con más posibilidades de reconocimiento social y retorno financiero.

Sin embargo, noto que en general trabajan duro, creo que tienen interés, tienen motivación. Hay aquella historia, preguntas el primer día por que han elegido Pedagogía, algunos dicen que no querían hacer Pedagogía, querían hacer Psicología, querían hacer esto o aquello; y en esta falta de opción han elegido Pedagogía; pero creo que nos toca a nosotros, con nuestra práctica en el día a día del curso, estimularlos, motivarlos, para que quieran ser profesores, algo que necesitamos mucho en el país. ¡Mucho! (Docente 5)

Compromiso: El profesor piensa que puede ayudar a aumentar el grado de compromiso de los alumnos con los aprendizajes (cinco extractos). 
Como en la categoría homónima, ubicada en el grupo de la Teoría Directa, aquí los profesores parecen opinar que la motivación tiene que ser algo interno a los alumnos, algo que poseen o no poseen. Pero, a diferencia del primer grupo, estos profesores creen que pueden intervenir de dos formas: en primer lugar, si el alumno no presenta esa motivación, a la que llaman "compromiso", los profesores deben intentar "recuperarla" de alguna manera. Y si el alumno presenta esa motivación, los profesores pueden, a su vez, partir de ella y reorientarla cuando sea necesario.

Creo que interfiere, ya que esta relación con el saber se vuelve una relación poco comprometida. Por eso creo que siempre es un desafio para el profesor comprender esta realidad, intentar buscar formas de atraerlos, de hacerles pensar. Es algo hecho con ellos, porque creo que aprendemos a partir de lo que ellos traen. (Docente 11)

Esfuerzo: El esfuerzo del alumno es un elemento importante para su aprendizaje, pero puede ser mejorado por lo que hace el profesor en clase (cuatro extractos).

De acuerdo con la teoría de atribuciones de Weiner (Schunk, Meece \& Pintrich, 2014), atribuir el resultado de acciones, éxitos o fracasos a factores inestables y controlables (como el esfuerzo), es preferible a atribuirlos a factores estables e incontrolables (como supuestamente sería la inteligencia). Las respuestas de esta subcategoría, en consonancia con la idea central de la concepción interpretativa sobre la motivación (que ésta se puede cambiar por medio de intervenciones), valoran el esfuerzo emprendido por sus estudiantes más que cualquier otro rasgo personal que pueda denotar estabilidad y/o imposibilidad de control. Pensar así tiene, de hecho, consecuencias positivas, pues puede llevar los profesores a intervenir en aumentar y dirigir el esfuerzo discente.

Creo que mientras más se esfuercen y se involucren más aprenderán. Entonces, si logramos que ellos se animen a eso, tienen un resultado de aprendizaje mucho mayor. (Docente 31) 
Factores relativos al alumno (contexto)

Siguen en este apartado las respuestas que hacen hincapié en el entorno de los alumnos como un factor importante en la determinación del interés por las actividades académicas y por la cantidad de esfuerzo invertido en ellas. Como es característico de la Teoría Interpretativa, esta influencia del contexto puede ser atenuada, o incluso cambiada, por las relaciones establecidas en el aula.

Condiciones socioeconómicas: Las condiciones socioeconómicas de los alumnos interfieren en su rendimiento académico (siete extractos).

Además del hecho de que los alumnos que acceden a los cursos de formación de profesores sean oriundos de una educación básica precaria y hayan elegido estos cursos por la facilitad de ingreso, otra característica importante de buena parte de estos estudiantes es que tienen que trabajar mientras estudian. Este alumno trabajador casi siempre es visto por sus profesores como alguien que trae consigo un obstáculo para aprender: la falta de tiempo para estudiar y para realizar las actividades fuera de la clase.

Para los profesores con concepciones directas, la falta de tiempo sería un obstáculo prácticamente insuperable. Para los de la Teoría Interpretativa, todavía, esta situación concreta puede estar lejos del ideal, pero se puede sacar más provecho del tiempo disponible.

Entonces, tenemos un alumnado que es un tanto trabajador, no son burgueses. Por la mañana y por la tarde, por la noche, hasta las diez de la noche, no hay cómo, sábado durante el día. ¿A qué horas estudian? Yo no lo sé. Tenemos ahí también un problema, pero diría que siempre puede ayudar, sí, desde la estructura física de los ambientes, pero no puede ser tan cómodo, pues pueden hasta dormirse [risas]. Sin embargo, la tecnología, buenas bibliotecas, creo que serían interesantes y obvios. El profesor tiene que salir un poco, tiene que hacer juegos, tiene que hacer dinámicas. (Docente 32) 
Factores relativos a la enseñanza (profesor)

Este es el grupo que contiene más extractos de respuestas sobre la motivación. Aunque tenga menos categorías que los factores relativos al alumno (personales) de la Teoría Directa, hay más extractos aquí ubicados que en todos los grupos clasificados como Teoría Directa. Como fue mencionado, las categorías aquí agrupadas son muy semejantes entre ellas, pues todas hacen hincapié en las maneras de actuar del profesor para cambiar los estados motivacionales de sus estudiantes. Lo que diferencia las categorías es el énfasis que pone cada entrevistado respecto de qué aspecto de la práctica docente estaría siendo más decisivo para la motivación.

Evaluación: El profesor busca maneras de evaluar que mejoren la relación del alumno con el proceso de aprendizaje (cinco extractos).

Mientras que la concepción directa entiende la evaluación como una oportunidad de distribuir refuerzos y castigos a la manera conductista, en la concepción interpretativa la evaluación es un momento importante en el proceso de aprendizaje, pues proporciona a los estudiantes un momento para reflexionar y también para aprender. Pero, aunque ya se pueda considerar un avance con relación a la concepción directa, las respuestas aquí ubicadas están lejos de una concepción más constructiva, como recomienda Huertas (2009): "No basta con evaluar el aprendizaje, hay que evaluar para el aprendizaje, para que el estudiante pueda seguir aprendiendo, idealmente casi ya solo por sí mismo" (itálicas en el original) (p. 175).

Reconsiderar la evaluación. No es solamente aumentar la exigencia, no es solamente eso. Hay profesores que creen que basta con hacer las correcciones en los trabajos de los alumnos y devolverlos. Creo que no es suficiente. Creo que es importante la devolución, una lectura cuidadosa. Pero nosotros estamos haciendo con los alumnos lo que los profesores de educación básica hacen con los suyos. El alumno no tiene una habilidad cuando llega en el primer año, nosotros no volvemos atrás para... Creo que eso es algo grave que está pasando. (Docente 15)

Profesor: La conducta del profesor en el aula y su relación con la docencia y con sus alumnos son decisivas para la relación de los alumnos con el aprendizaje (11 extractos). 
Las respuestas que aparecen aquí fueron dadas por profesores que entienden que la actitud de los alumnos hacia el aprendizaje depende de sus propias actitudes como docentes. Para estos profesores no basta con exigir de sus alumnos esfuerzo y dedicación. Es imprescindible demostrar que se está comprometido con la docencia, para que eso pueda, de alguna manera, contagiar a los alumnos.

El currículo y el compromiso de los profesores que llevan el curso, creo que eso contribuye para la mayor parte de ese empeño [de los alumnos], independientemente de la relación que tenemos con el mercado laboral. (Docente 28)

Clase: Lo que se hace en el aula favorece la relación del alumno con lo que debe ser aprendido (seis extractos).

Los profesores con respuestas de este tipo entienden que el clima motivacional de la clase interfiere en el desempeño de los alumnos, tal como defienden Alonso-Tapia, Ruiz y Huertas (2020). Pero, de manera distinta de lo que recomienda la literatura específica sobre este tema (Huertas, 2009), estos profesores no hacen cambios significativos en el diseño, presentación y evaluación de las actividades didácticas que promuevan el interés, la autonomía y la autorregulación, por ejemplo.

Me siento obligado a hacer una clase más dialógica, pienso que si en psicología de la educación, si la clase no es dialógica, si no es un espacio para que todos hablen, si no es un espacio de escucha para todos, es como si yo estuviera, no lo sé, yendo en contra de lo más básico de la psicología de la educación, una asignatura que trata de los "aspectos subjetivos del espacio de la clase". (Docente 27)

Estrategias: El profesor elige estrategias para mejorar la relación de los alumnos con el aprendizaje y su desempeño (12 extractos).

En esta categoría los profesores parecen reconocer más claramente su rol en el aula: crear un contexto favorable para que los estudiantes puedan dedicarse de manera interesada y persistente a las tareas de aprendizaje (Alonso-Tapia, Ruiz \& Huertas, 2020). 
Los entrevistados se ven como responsables de intervenir en los estados motivacionales de sus alumnos, buscando soluciones para los problemas que disminuyen el esfuerzo hacia el aprendizaje.

Si tienes un grupo que no demuestra ese esfuerzo, tu esfuerzo tendrá que ser mucho más grande, porque vas a tener que movilizar el esfuerzo del grupo. Pensar en estrategias para ayudar a esa motivación que, a veces, vas a necesitar trabajar con la motivación extrínseca, vas a necesitar crear algunas estrategias para que haya un interés y por el tema que estás tratando. Entonces, esos temas interfieren mucho en mi manera de ser profesora. (Docente 31)

Factores relativos a la enseñanza (currículo)

Además de lo que se lleva a cabo en el aula, los entrevistados creen que las estructuras, tanto físicas como conceptuales, de las cuales forman parte los alumnos, interfieren en su motivación y consecuente desempeño. Aunque una de las categorías de este grupo lleve de manera más restrictiva el título de "currículo", refiriéndose a la forma de organizar las disciplinas y los contenidos, se extenderá ese concepto a la ordenación del espacio físico, horarios y materiales disponibles para el aprendizaje (Gimeno Sacristán, 2015).

Currículo: El currículo interfiere en la relación de los alumnos con el aprendizaje y con el desempeño (cuatro extractos).

Aquí se habla de "currículo" en su acepción más restringida, que es la de un conjunto ordenado de disciplinas distribuidas a lo largo de un curso o carrera. El énfasis de estas respuestas está justamente en la organización curricular como promotora de la motivación. Estos entrevistados no manejan aún la idea de un currículo que ayude a desarrollar la autonomía o las competencias del aprender a aprender (Lluch Molins y Portillo Vidiella, 2018).

Creo que la organización del currículo, por ejemplo, esas críticas que nosotros tenemos al currículo actualmente han favorecido de alguna manera ese esfuerzo de los estudiantes. Creo que eso lo favorece. (Docente 28) 
Estructura: La estructura ofrecida a los estudiantes, como aulas y horarios, interfiere en sus aprendizajes (tres extractos).

En esta otra categoría del grupo de los factores curriculares se adopta una concepción un poco más amplia de currículo, incluyendo el tiempo y el espacio en el que ocurren las clases. Los profesores que mencionaron estos aspectos entienden que el contexto de aprendizaje va más allá de las actividades propuestas por los docentes en aula. Este contexto está constituido por dónde y cuándo suceden las clases.

Creo que simbólicamente el aula representa un espacio malo para quedarse; sin ventilación, sin luminosidad... Luego pensé, tenemos un espacio a las afueras de la universidad, una fila de eucaliptos, tienen un olor maravilloso, un sol fantástico... Pensé en comprar unas esterillas, distribuir, para estar afuera. Sin embargo, cuarenta y tres alumnos, no hay cómo, y eso también es un problema, la cantidad de alumnos en clase, obligados a estar allí. (Docente 25)

\subsubsection{Resultados cualitativos de la Teoría Constructiva}

Solo 11 extractos (de un total de 117) fueron categorizados como representativos de la Teoría Constructiva. Con estos extractos se formaron dos subcategorías, pertenecientes a dos grupos distintos de factores: uno relativo a la enseñanza, centrado en el profesor, que agrega subcategorías de las tres teorías, y otro grupo relativo a la enseñanza, centrado en el currículo, compuesto por respuestas de las teorías Constructiva e Interpretativa.

Factores relativos a la enseñanza (profesor)

La única subcategoría ubicada en este grupo, como se puede inferir de las definiciones precedentes, está más cerca de la Teoría Interpretativa que de la Teoría Directa, ya que mantiene la idea fundamental de que el profesor puede hacer algo para cambiar los estados motivacionales de sus alumnos. Pero, a diferencia de los casos anteriores, las respuestas que aparecen aquí se centran en la importancia del aprendizaje para los procesos motivacionales. 
Método: El método, es decir, las decisiones tomadas por el profesor y las acciones llevadas a cabo por él deben tener como objetivo el aprendizaje de los alumnos (cinco extractos).

De acuerdo con Huertas (2006), "está comprobado que cuánto más éxito obtenga una persona, ésta experimentará una mayor motivación. Por el contrario, si se acumulan los fracasos, las expectativas de éxito disminuyen trágicamente" (p. 253). Los profesores que dieron las respuestas recogidas a continuación parecen comprender y adoptar ese principio, pues buscan garantizar el aprendizaje de sus estudiantes incluso como una manera de promover su motivación.

Es cuando intento siempre pensar en metodologías más activas $y$, de esta manera, intento ser coherente con lo que estudio, las teorías, y lo que tomo como relevante de las teorías sobre enseñanza-aprendizaje e intento ser coherente dentro de lo que, para mí, tiene que ser enseñanza y aprendizaje. Darle oportunidad al alumno a participar, que el aprendizaje sea significativo. (Docente 26)

Factores relativos a la enseñanza (currículo)

La categoría aquí ubicada agrupa respuestas que representan la esencia de la Teoría Constructiva, que entiende el aprendizaje como una construcción de sentido por parte del aprendiz.

Sentido: El currículo académico debe tener sentido para los estudiantes, para que estos puedan involucrarse (seis extractos).

De acuerdo con Pozo (2008), las teorías constructivistas "vinculan el aprendizaje al significado que el aprendiz atribuye a los ambientes a los que se enfrenta, en función de las estructuras cognitivas y conceptuales desde las que interpreta ese ambiente" (p. 141). Aunque nuestros entrevistados hablen de "sentido" y no de "significado", como señala Pozo, se sabe que hay una relación estrecha entre estos dos términos, pudiéndose incluso utilizarlos como sinónimos a depender del contexto. Así, se puede inferir que los profesores de este grupo ya se acercan en algunos momentos (o en algunos aspectos de sus prácticas pedagógicas) a la idea constructiva de enseñanza y aprendizaje. 
Estudiar es bueno, pero eso implica que tengo esfuerzo, desprendo energía, renuncio a algunas cosas para establecer prioridades. Entonces, el interés influye en eso, porque cuando el alumno comprende el sentido y asume para sí mismo, entonces las dificultades se convierten mucho más en un desafío que en un muro de lamentaciones. (Docente 6)

\section{Conclusiones}

Este estudio tuvo como objetivo analizar las teorías implícitas de los profesores acerca de la motivación de sus alumnos para aprender en sus asignaturas. Sobre esto, los datos cuantitativos demuestran la misma tendencia de otros estudios (Alves y Pozo, 2014; Pozo, Loo y Martín, 2016; Scheuer y Pozo, 2006), esto es, que los sujetos dan, en su mayoría, respuestas clasificadas como "interpretativas".

Respecto de los datos cualitativos, hay dos grandes conjuntos de explicaciones, con características un poco distintas. El primer conjunto se puede llamar "factores relativos al alumno", que incluye aspectos personales y de su entorno o contexto. Es en el que se ubica la mayor parte de las subcategorías, todas ellas pertenecientes a la Teoría Directa y a la Teoría Interpretativa. El segundo conjunto se puede denominar "factores relativos a la enseñanza", e incluye aspectos relacionados tanto con los docentes como con el currículo. En este grupo, aunque la mayoría de las subcategorías sean de la Teoría Interpretativa, hay representantes de la Teoría Directa y de la Teoría Constructiva en igual cantidad.

Esta distribución está, en parte, reforzada por la prueba de Spearman, que ha evidenciado la tendencia de los profesores con respuestas clasificadas como Teoría Directa a responsabilizar a las características de los estudiantes por su propia motivación; así como la tendencia de los docentes con concepciones pertenecientes a la Teoría Interpretativa de incluir el contexto de aprendizaje como factor influyente en la motivación discente.

Sobre esta manera de interpretar estos dos elementos cruciales para el proceso educativo, que son el alumno y la enseñanza, se puede decir que, al hablar de los primeros, los profesores lo hacen 
a partir de concepciones más sencillas sobre la motivación y, por tanto, más alejadas de lo que afirman los hallazgos teóricos más actuales en este campo de estudios. Estas concepciones ya permiten a los entrevistados pensar la motivación del estudiante como un proceso que puede mejorar con la intervención docente (Teoría Interpretativa), pero en muchas situaciones hace pensar aun que la motivación es algo inherente al alumno y que este debe poseerla para que haya éxito en el aprendizaje universitario.

Se puede afirmar que los profesores cuya mayoría de respuestas se congregó en estas dos teorías implícitas se acercan más al primer polo de los cuatro factores sobre la motivación, expuestos por AlonsoTapia (2005) y comentados anteriormente. Es decir, estos docentes tienden a pensar, en mayor o menor grado, que la motivación es un problema de la voluntad del estudiante; que puede variar si se cambia un aspecto del entorno; que es estática y que se consigue con intervenciones puntuales.

Cuando hablan de la enseñanza, los entrevistados presentan ya teorías un poco más elaboradas acerca del rol de sus acciones didácticas y del currículo en la motivación discente. Hay una mayor cantidad relativa de subcategorías clasificadas como Teoría Interpretativa y una sensible disminución de las subcategorías del tipo Teoría Directa. Además de eso, aparecen en los factores relativos a la enseñanza dos subcategorías clasificadas como Teoría Constructiva, que no había en los factores relativos al alumno.

Estas teorías se acercan más al segundo polo de los propuestos por Alonso-Tapia (2005). Esto significa que los profesores que dieron estas respuestas tienden a pensar la motivación como un resultado de la interacción entre el alumno y el entorno; que cambia si se actúa sobre el clima de aprendizaje; que es dinámica y que requiere una intervención continua.

La calidad de la educación depende, entre otros factores, de la calidad del aprendizaje. Para que esto último ocurra es necesario tener aprendices con cierto grado de motivación y eso depende en 
gran medida de lo que hacen los profesores en las aulas. A su vez, la práctica de los docentes depende de las teorías que mantengan, aunque teoría y práctica no siempre coincidan, pues parece que la primera está siempre en un nivel más elaborado que la última (Pozo, Loo y Martín, 2016).

En suma, en la búsqueda de una mejora en la calidad de la educación, por la vía de la motivación y del aprendizaje, habría que seguir las recomendaciones de Alonso-Tapia, Ruiz y Huertas (2020), en el sentido de ayudar los docentes a construir una concepción explícita sobre la motivación más elaborada, pero también una práctica educativa que esté de acuerdo con esa concepción. Juntas, teoría y práctica deben tener como reto un alumno cada vez más dedicado y autónomo frente a los desafíos de la formación académica.

\section{Referencias}

Alonso-Tapia, J. (2005). Motivar en la escuela, motivar en la familia. Madrid: Santillana.

Alonso-Tapia, J., Ruiz, M. Á. \& Huertas, J. A. (2020). Differences in classroom motivational climate: causes, effects and implications for teacher education. A multilevel study. Anales De Psicologia/ Annals of Psychology, 36(1), 122-133. DOI: https://doi.org/10.6018/ analesps.337911" https://doi.org/10.6018/analesps.337911

Alves, I. P. y Pozo, J. I. (2014). Las concepciones implícitas de los profesores universitarios sobre los requisitos para el aprendizaje. Revista da FAEEBA: Educação e Contemporaneidade, 41, 191-203.

Bardin, L. (2002). Análisis de Contenido. Madrid: Ediciones Akal.

Errázuriz-Cruz, M. (2020). Teorías implícitas sobre escritura de estudiantes y formadores de pedagogía. Magis, Revista Internacional de Investigación en Educación, 13, 1-26. DOI: https://doi.org/10.11144/Javeriana.m13.tiee

García, G. M. y Martínez, G. M. F. (2013). La evaluación continua, un incentivo que incrementa la motivación para el aprendizaje. Revista Iberoamericana de Evaluación Educativa, 6(2), 265-278.

Gimeno Sacristán, J. (2015). Los contenidos: una reflexión necesaria. Madrid: Morata. 
Han, J. \& Yin, H. (2016). Teacher motivation: Definition, research development and implications for teachers, Cogent Education, 3: 1. DOI: https://doi.org/10.1080/2331186X.2016.1217819

Huertas, J. A. (2006). Motivación: querer aprender. Buenos Aires: Aique Grupo Editor.

Huertas, J. A. (2009). Aprender a fijarse metas: Nuevos estilos motivacionales. En J. I. Pozo y M. del P. Pérez Echeverría (Coords.), Psicología del aprendizaje universitario: La formación en competencias (pp. 164-181). Madrid: Morata.

Lluch Molins, L. y Portillo Vidiella, M. C. (2018). La competencia de aprender a aprender en el marco de la educación superior. Revista Iberoamericana de Educación, 78(2), 59-76. DOI: https://doi.org/10.35362/rie7823183

Martín, E., Mateos, M., Martínez, P., Cervi, J., Pecharromán, A. y Villalón, R. (2006). Las concepciones de los profesores de educación primaria sobre la enseñanza y el aprendizaje. En J. I. Pozo, N. Scheuer, M. P. Pérez Echeverría, M. Mateos, E. Martín y M. de la Cruz. (Orgs.), Nuevas formas de pensar la enseñanza y el aprendizaje: Las concepciones de profesores y alumnos (pp. 171-188). Barcelona: Graó.

Mateos, M. (2009). Aprender a leer textos académicos: más allá de la lectura reproductiva. En J. I. Pozo y M. del P. Pérez Echeverría (Coords.), Psicología del aprendizaje universitario: la formación en competencias (pp. 106-119). Madrid: Morata.

Pérez Echeverría, M. P., Mateos, M., Scheuer, N. y Martín, E. (2006). Enfoques en el estudio de las concepciones sobre el aprendizaje y la enseñanza. En J. I. Pozo, N. Scheuer, M. P. Pérez Echeverría, M. Mateos, E. Martín, M. de la Cruz. (Orgs.), Nuevas formas de pensar la enseñanza y el aprendizaje: Las concepciones de profesores y alumnos (pp. 95-134). Barcelona: Graó.

Pozo, J. I. (2008). Aprendices y Maestros: La psicología cognitiva del aprendizaje. Madrid: Alianza Editorial.

Pozo, J. I. (2009). Adquirir una concepción compleja del conocimiento: creencias epistemológicas y concepciones de aprendizaje. En J. I. Pozo y M. P. Pérez Echeverría (Coords.), Psicología del aprendizaje universitario: La formación en competencias (pp. 70-86). Madrid: Morata.

Pozo, J. I. y Scheuer, N. (1999). Las concepciones sobre aprendizaje como teorías implícitas. En J. I. Pozo y C. Monereo (Coords.), El aprendizaje estratégico (pp. 87-108). Madrid: Aula XXI/Santillana.

Pozo, J. I., Scheuer, N., Mateos, M. y Pérez Echeverria, M. del P. (2006). Las teorías implícitas sobre el aprendizaje y la enseñanza. En J. I. Pozo, 
N. Scheuer, M. P. Pérez Echeverría, M. Mateos, E. Martín y M. de la Cruz. (Orgs.), Nuevas formas de pensar la enseñanza y el aprendizaje: Las concepciones de profesores y alumnos (pp. 95-134). Barcelona: Graó.

Pozo, J. I., Loo, C. y Martín, E. (2016). El cambio de las concepciones y las prácticas docentes como factor de cambio educativo. En J. Manzi y R. M. García, (Eds.) Abriendo las puertas del aula: nuevos enfoques para la transformación de las prácticas docentes (pp. 545-584). Santiago de Chile: PUC.

Scheuer, N. y Pozo, J. I. (2006). ¿Qué cambia en las teorías implícitas sobre el aprendizaje y la enseñanza? Dimensiones y procesos del cambio representacional. En J. I. Pozo, N. Scheuer, M. P. Pérez Echeverría, M. Mateos, E. Martín y M. de la Cruz. (Orgs.), Nuevas formas de pensar la enseñanza y el aprendizaje: Las concepciones de profesores y alumnos (pp. 375-402). Barcelona: Graó.

Schunk, D. H., Meece, J. y Pintrich, P. (2014). Motivation in Education: Theory, Research and Applications. USA: Pearson.

Torrado, J. A. y Pozo, J. I. (2006). Del dicho al hecho: De las concepciones sobre el aprendizaje a la práctica de la enseñanza de la música. En J. I. Pozo, N. Scheuer, M. P. Pérez Echeverría, M. Mateos, E. Martín y M. de la Cruz. (Orgs.), Nuevas formas de pensar la enseñanza y el aprendizaje: Las concepciones de profesores y alumnos (pp. 205-230). Barcelona: Graó.

Recibido: 07/07/2020

Aceptado: 19/10/2020 